\title{
An axiomatic definition of divergence for intuitionistic fuzzy sets
}

\author{
Ignacio Montes ${ }^{1}$ Vladimir Janiš ${ }^{2}$ Susana Montes ${ }^{1}$ \\ $1 \quad$ University of Oviedo, Spain \\ imontes@uniovi.es montes@uniovi.es \\ 2 Matej Bel University, Slovak Republic \\ vladimir.janis@umb.sk
}

\begin{abstract}
An axiomatic definition of divergence measure for intuitionistic fuzzy sets (IFSs, for short) is presented in this work, as a particular case of dissimilarity between IFSs. As the concept of divergence measure is more restrictive, it has particular properties which are studied. Furthermore, the relationships among IF-divergences, dissimilarities and distances are studied. We also provide some methods for building divergence measure for IFSs. They will allow us to conclude this work with a classification of the usual functions used in the literature for measuring the difference between intuitionistic fuzzy sets in two classes: which are divergence measures between IFSs and which are not.
\end{abstract}

Keywords: Intuitionistic fuzzy set, divergence measure, dissimilarity measure, distance.

\section{Introduction}

Since Zadeh introduced fuzzy sets in 1965 (see [25]), this theory has become very popular. We can find in the literature many papers and journals that focus on fuzzy sets. In particular, they have been used to model some real situations were crisp theory incurs in a high lost of information.

Since the comparison of descriptions of two objects is necessary in many fields as psychology, analogy, physical sciences, image processing, clustering, deductive reasoning, case-based reasoning, etc., to measure the difference between fuzzy sets has been an objective of many studies. For example, Bouchon et al. ([4]) proposed a general axiomatic that model all the types of functions used for measuring that difference. Montes et al. ([21]) made an axiomatic definition of a divergence measure. Furthermore, Couso et al. ([8]) used the concepts of dissimilarity to measure the difference between fuzzy random variables. Usually, the comparison is achieved through a measure intended to determine to which extent the descriptions have common points or differ from each other (for a general proposal see [4]).

Although fuzzy sets are very useful in many context, Atanassov proposed a more general theory (see [1]), based on intuitionistic fuzzy sets. If fuzzy sets allow a degree of membership, intuitionistic fuzzy sets allow a degree of membership and a degree of non-membership. This type of sets has shown to be appropriate to model a lot of situations in which fuzzy sets do not consider all the available information. This theory is currently being developed, and the proof is the amount of literature we can find (see for example $[2,3,5,9,15]$ ).

For measuring the difference between intuitionistic fuzzy sets, we can find an axiomatic definition of dissimilarity measures and several examples (see, for instance, $[13,14,11,17,19,22,23])$. In this work, we proposed an axiomatic definition of a divergence measure for intuitionistic fuzzy sets as a particular case of dissimilarity measure with specific properties. As we will prove, this notion is more restrictive than dissimilarity, and this allows more variety of properties. Our aim is to study the relationships among three ways of measuring the difference between intuitionistic fuzzy sets: the classical notion of distances, dissimilarities and divergences. We also want to study some properties of the notion of divergence.

This work is organized as follows: Section 2 is used for introducing some well-known concepts. In the first part we will recall the notion and properties of intutionistic fuzzy sets. Then we will recall the axiomatic definition of divergence for fuzzy sets, which will be our starting point in next section. Thus, in Section 3 we will propose an axiomatic definition of divergence for intuitionistic fuzzy sets. We are going to study the relationships among IF-divergences, dissimilarities and distances, and we will show some methods for building IFdivergences. Section 4 is devoted to prove if some of the most usual dissimilarities measures are or not IF-divergences. We conclude the work with some final comments and open problems.

\section{Preliminaries}

This section is devoted to introducing some wellknown concepts, and also fixing the notation. First of all, we will focus on the theory of intuitionistic fuzzy sets. Then, we will recall the axiomatic definition of a divergence measure for fuzzy sets. 


\subsection{Intuitionistic Fuzzy Sets}

Fuzzy sets are used to model a lot of real problems. However, there are other situations where the model can be improved. Atanassov introduced intuitionistic fuzzy sets to model situations in which every point in the universe has a degree a membership, a degree of non-membership and a degree of indeterminacy or uncertainty. In this way he defined an intuitionistic fuzzy set as follows:

$$
A=\left\{\left(x, \mu_{A}(x), \nu_{A}(x)\right) \mid x \in X\right\},
$$

where $\mu_{A}$ and $\nu_{A}$ are functions $\mu_{A}, \nu_{A}: X \rightarrow[0,1]$ such that $0 \leq \mu_{A}(x)+\nu_{A}(x) \leq 1$.

On this way, $\mu_{A}$ and $\nu_{A}$ denote the degree of membership and non-membership, respectively. Thus, the function $\pi_{A}(x)=1-\mu_{A}(x)-\nu_{A}(x)$, called the intuitionistic fuzzy index or the hesitation index, expresses lack of knowledge of whether $x$ belongs to $A$ or not.

Since intuitionistic fuzzy sets allow two degrees of freedom into a set description, and fuzzy sets only allow one, this generalization gives us an additional possibility to represent the lack of information what leads when we try to describe many real problems. Thus, they are very convenient to model situations where human answers are present: yes, no or does not apply. A good example of these kind of situations is voting, since human voters can be divided into three groups: vote for, vote against or abstain (see for example [22]).

From now on, IFSs $(X)$ will denote the set of all intuitionistic fuzzy sets on $X$. Given $A, B \in$ $\operatorname{IFS} S(X)$, the operations of union, intersection and complement and the inclusion relation can be defined as follows:

- Union of every $A$ and $B$ :

$$
A \cup B=\left\{\left(x, \mu_{A \cup B}(x), \nu_{A \cup B}(x)\right) \mid x \in X\right\},
$$

where $\mu_{A \cup B}(x)=\max \left\{\mu_{A}(x), \mu_{B}(x)\right\}$ and $\nu_{A \cup B}(x)=\min \left\{\nu_{A}(x), \nu_{B}(x)\right\}$.

- Intersection of every $A$ and $B$ :

$$
A \cap B=\left\{\left(x, \mu_{A \cap B}(x), \nu_{A \cap B}(x)\right) \mid x \in X\right\},
$$

where $\mu_{A \cap B}(x)=\min \left\{\mu_{A}(x), \mu_{B}(x)\right\}$ and $\nu_{A \cap B}(x)=\max \left\{\nu_{A}(x), \nu_{B}(x)\right\}$.

- Complement of every $A$ :

$$
A^{c}=\left\{\left(x, \nu_{A}(x), \mu_{A}(x)\right) \mid x \in X\right\} .
$$

- $A$ is a subset of $B$ (denoted by $A \subseteq B$ ) if and only if for every $x \in X$ it holds that:

$$
\mu_{A}(x) \leq \mu_{B}(x) \text { and } \nu_{A}(x) \geq \nu_{B}(x) .
$$

We have considered the initial definitions given by Atanassov ([1, 2]), since they have been the most usually considered in the literature. However, let us recall that some generalizations based on t-norms and t-conorms have already been presented (see for example [10]).

\subsection{Divergences for Fuzzy Sets}

As we commented previously, a fuzzy set can be seen as a particular case of intuitionistic fuzzy set. In this particular context, several measures have been proposed in order to measure the degree of difference between two fuzzy sets. A general study was presented in [4]. As a particular case was widely studied in [21]. There, an axiomatic definition of a divergence measure for fuzzy sets was introduced. It was based on the following natural properties:

- It is a nonnegative and symmetric function of the two fuzzy sets.

- The divergence of a fuzzy sets with itself is zero.

- The "more similar" two fuzzy sets are, the lower divergence between them.

These properties were mathematically described in the following way.

Definition 2.1 ([21]) Let us consider an universe $X$, and let $F S(X)$ denotes the set of all fuzzy sets on $X$. A map $D: F S(X) \times F S(X) \rightarrow \mathbb{R}$ is a divergence measures if for every fuzzy sets $A$ and $B$ it fulfills the following conditions:

1. $D(A, B)=D(B, A)$.

2. $D(A, A)=0$.

3. $D(A \cap C, B \cap C) \leq D(A, B)$, for every $C \in$ $F S(X)$.

4. $D(A \cup C, B \cup C) \leq D(A, B)$ for every $C \in$ $F S(X)$.

The nonnegativity of the divergence is not required in the previous axioms. However, it is trivial to deduce it from axioms 2 and 3 (or 2 and 4).

Montes et al. ([21]) studied some properties of divergences, and they also proved that the most of the usual functions used in the literature for measuring the difference between fuzzy sets are, in fact, divergences.

Example 2.2 ([21]) Let us consider a finite universe $X$. The following functions are divergence measures for fuzzy sets.

- $D_{1}(A, B)=S_{\mathrm{x} \in \mathrm{X}}(|A(x)-B(x)|)$, for every $A, B \in F S(X)$, where $S$ is a t-conorm.

- In particular, if the t-conorm is the sum $(S(x, y)=x+y)$, we obtain $D_{2}(x, y)=$ $\sum_{\mathrm{x} \in \mathrm{X}}|A(x)-B(x)|$. Thus, we are measuring the difference between fuzzy sets using the Hamming distance between fuzzy sets.

- $D_{3}(A, B)=\sum_{\mathrm{x} \in \mathrm{X}} h(A(x), B(x))$, where $h(t, z)=g\left(\frac{t+z}{2}\right)-\frac{g(t)+g(z)}{2}$ and $g:[0,1] \rightarrow \mathbb{R}^{+}$ is a concave function, increasing in $[0,0.5]$, symmetric with respect to the point $\frac{1}{2}$ and $g(0)=g(1)=0$.

This particular idea for fuzzy sets is our starting point in this paper, in order to introduce an axiomatic definition of comparison measure between IFSs. 


\section{Divergence measures for Intuitionistic Fuzzy Sets}

This section is devoted to introduce an axiomatic definition of a measure of comparison of two intuitionistic fuzzy sets. In the first part we will introduce the axioms and we are going to study the relationships among IF-divergences, dissimilarities and distances. Then, we will introduce some basic properties and some methods for building IFdivergences.

\subsection{Axiomatic definition}

Some many functions have been presented in the literature with the aim of measuring the difference between intuitionistic fuzzy sets. The most common ones were dissimilarities. Let us recall that a dissimilarity measure for intuitionistic fuzzy sets is a function $D_{I S}$ from $\operatorname{IFSs}(X) \times I F S s(X)$ to $\mathbb{R}$ such that for every $A, B, C \in I F S s(X)$, it fulfills the following properties:

Ax.1 $D_{I S}(A, B)=D_{I S}(B, A)$.

Ax.2 $D_{I S}(A, A)=0$.

$\widetilde{\mathrm{Ax} .3} A \subset B \subset C$, then

$$
\begin{aligned}
& D_{I S}(A, C) \geq D_{I S}(A, B) . \\
& D_{I S}(A, C) \geq D_{I S}(B, C) .
\end{aligned}
$$

In next section we will enumerate several examples of dissimilarities, presented in the literature. However, some of them have some drawback. Thus, we have considered a particular case of dissimilarities in order to obtain stronger properties for the measure. Thus, we consider the concept of divergence measure for intuitionistic fuzzy sets, which is axiomatically defined as follows:

Definition 3.1 Let $X$ be an universe, and let $I F S s(X)$ denote the set of all intuitionistic fuzzy sets on $X$. A map $D_{\mathrm{IF}}: \operatorname{IFSs}(X) \times \operatorname{IFSs}(X) \rightarrow$ $\mathbb{R}$ is a divergence measure for intuitionistic fuzzy sets (IF-divergence, for short) if for every $A, B \in$ IFSs $(X)$ it fulfills the following properties:

$A x .1 D_{\mathrm{IF}}(A, B)=D_{\mathrm{IF}}(B, A)$.

Ax.2 $D_{\mathrm{IF}}(A, A)=0$.

$A x .3 D_{\mathrm{IF}}(A \cap C, B \cap C) \leq D_{\mathrm{IF}}(A, B)$ for every $C \in I F S s(X)$.

$A x .4 D_{\mathrm{IF}}(A \cup C, B \cup C) \leq D_{\mathrm{IF}}(A, B)$ for every $C \in I F S s(X)$.

In Section 4 we will provide some examples of dissimilarity measures which are not IF-divergences. The following result will be useful to prove that every IF-divergence is a dissimilarity measure.

Lemma 3.2 Let us consider the intuitionistic fuzzy sets $A, B, C$ and $D$ on $X$ such that $A \subset C \subset D \subset$ $B$. Then it holds that $D_{\mathrm{IF}}(A, B) \geq D_{\mathrm{IF}}(C, D)$.

Using this lemma we can prove the following proposition.
Proposition 3.3 Every IF-divergence is also a dissimilarity measure for intuitionistic fuzzy sets.

Thus, we can see that the definition of IFdivergences is more restrictive than the definition of dissimilarities.

Another important way of comparing two IFSs was by means of distances. Let us note that there is not a general relationship between IF-divergences and distances. To see that, let us consider the map $d$ defined on the real interval $[0,1]$ as follows:

$$
d(x, y)= \begin{cases}|x-0.2| & \text { if } x \notin\{0.1,0.2\}, y=0.1 . \\ |x-0.1| & \text { if } x \notin\{0.1,0.2\}, y=0.2 . \\ |0.2-y| & \text { if } x=0.1, y \notin\{0.1,0.2\} . \\ |0.1-y| & \text { if } x=0.2, y \notin\{0.1,0.2\} . \\ |x-y| & \text { otherwise. }\end{cases}
$$

It is easy to check that $d$ is a metric on $[0,1]$. Therefore, for an universe $X=\{x\}$, the map

$$
d^{*}(A, B)=d\left(\left|\mu_{A}(x)-\mu_{B}(x)\right|,\left|\nu_{A}(x)-\nu_{B}(x)\right|\right)
$$

is a metric on $\operatorname{IFSs}(X)$. However, $d^{*}$ is not an IF-divergence, since for the intuitionistic fuzzy sets $A=\{(x, 0,0)\}, B=\{(x, 0.2,0)\}, C=\{(x, 0.15,0)\}$ and $D=\{(x, 0.18,0)\}$, it holds that

$$
\begin{aligned}
d^{*}(C \cup D, B \cup D) & =d^{*}(D, B)=0.08 \\
& >0.05=d^{*}(C, B) . \\
d^{*}(A \cap C, B \cap C) & =d^{*}(A, C)=0.15 \\
& >0.1=d^{*}(A, B) .
\end{aligned}
$$

Conversely, we can find an IF-divergence that is not a distance. For this aim it is enough to consider an universe $X=\{x\}$ and the map defined on $\operatorname{IFSs}(X)$ by:

$$
\begin{aligned}
& D_{\mathrm{IF}}(A, B)= \\
& \left(\mu_{A}(x)-\mu_{B}(x)\right) \log _{2}\left(\frac{\mu_{A}(x)\left(1-\mu_{B}(x)\right)}{\mu_{B}(x)\left(1-\mu_{A}(x)\right)}\right) .
\end{aligned}
$$

It can be proved that this function is an IFdivergence. Furthermore, if we consider the intuitionistic fuzzy sets $A=\{(x, 0.2,0)\}, B=$ $\{(x, 0.15,0)\}$ and $C=\{(x, 0.1,0)\}$, they do not fulfill the triangle inequality:

$$
\begin{aligned}
D_{\mathrm{IF}}(A, B)+D_{\mathrm{IF}}(B, C) & =0.058 \\
& <0.117=D_{\mathrm{IF}}(A, C) .
\end{aligned}
$$

We have just seen that there is not a general relationship between distances and IF-divergences.

To complete the relationships among distances, IF-divergences and dissimilarities, we are going to see that a distance is not necessarily a dissimilarity. For this aim, it is enough to consider once more the distance $d^{*}$ defined in Equation 1, and the intuitionistic fuzzy sets $A=\{(x, 0,0)\}, B=\{(x, 0.18,0)\}$ and $C=\{((x, 0.2,0)\}$. Then, we have that $A \subset$ $B \subset C$, but:

$$
\begin{aligned}
d^{*}(A, C) & =d(0.2,0)=0.1 \\
& <0.18=d(0.18,0)=d^{*}(A, B),
\end{aligned}
$$


and that contradicts the third axiom of dissimilarities.

In Figure 1 we can see summarized the relationships we have obtained among distances, IFdivergences and dissimilarities. A missed arrow means that this implication does not hold in general.

\section{IF-divergence $\longrightarrow$ Dissimilarity \\ Distance}

Figure 1: Relationships among distances, IFdivergences and dissimilarities.

\subsection{Basic properties}

In the previous subsection we have proposed an axiomatic definition of divergence measures for intuitionistic fuzzy sets as a particular case of dissimilarity measures. Next, we are going to study the specific properties that they fulfill. First, we recall that one of the reasonable properties that were required to be a good measure of the difference was the nonnegativity. It has not been imposed on the definition, since it can be easily obtained from the axioms 1 and 2 .

Proposition 3.4 Every IF-divergence is nonnegative.

A property that an IF-divergence $D_{\mathrm{IF}}$ can fulfill is:

$\widetilde{\mathrm{Ax} .5} D_{\mathrm{IF}}(A, B)=D_{\mathrm{IF}}\left(A^{c}, B^{c}\right)$, for every $A, B \in$ IFSs $(X)$.

This property can be fulfilled by some IFdivergence. However, we can find IF-divergences that do not fulfill it. For example, the IF-divergence $D_{\mathrm{IF}}$ defined in Equation 2. If we consider $A=$ $\{(x, 0.6,0.4)\}$ and $B=\{(x, 0.5,0.1)\}$, it holds that:

$$
\begin{aligned}
D_{\mathrm{IF}}(A, B) & =0.1 \log _{2}(1.5)=0.0585 \\
& \neq 0.7755=0.3 \log _{2}(6)=D_{\mathrm{IF}}\left(A^{c}, B^{c}\right) .
\end{aligned}
$$

In Section 4 we will see some examples of IFdivergences that fulfill this property.

The following proposition relates axioms 3 and 4 of the Definition 3.1 when the IF-divergence fulfills axiom 5 .

Proposition 3.5 Let $D_{\mathrm{IF}}$ be an IF-divergence. If $D_{\mathrm{IF}}(A, B)=D_{\mathrm{IF}}\left(A^{c}, B^{c}\right)$ for every $A, B \in$ IFSs $(X)$, then axioms 3 and 4 of Definition 3.1 are equivalents.

This result can be very useful when we are trying to proof if a function $D_{\mathrm{IF}}$ is or not an IF-divergence, because if such function fulfills axiom 1, 2 and 5, then axioms 3 and 4 are equivalents.

Using axioms 3 and 4, we can prove the following proposition.
Proposition 3.6 Let $A, B, C$ and $D$ be intuitionistic fuzzy sets on $X$. If $\mu_{C}=\mu_{D}$, then $D_{\mathrm{IF}}(A, B) \geq$ $D_{\mathrm{IF}}(C, D)$.

Remark 3.7 Applying the axioms of symmetry of the IF-divergences, it also holds that for every $A, B, C, D \in I F S s(X)$ such that $\nu_{C}=\nu_{D}$ then $D_{\mathrm{IF}}(A, B) \geq D_{\mathrm{IF}}(C, D)$.

To conclude this section, we are going to see two methods to built an IF-divergence. The first one is based on a divergence measure, and the second one is used to built IF-divergences from other IFdivergences.

Proposition 3.8 Let $X$ be an universe, and Let $D$ be a divergence for fuzzy sets on $X$. If $f$ is a map $f:[0, \infty) \times[0, \infty) \rightarrow[0, \infty)$ such that

1. $f(0,0)=0$, and

2. $f(\cdot, t)$ and $f(t, \cdot)$ are non-decreasing,

then the function $D_{\mathrm{IF}}$, defined by:

$$
D_{\mathrm{IF}}(A, B)=f\left(D\left(\mu_{A}, \mu_{B}\right), D\left(\nu_{A}, \nu_{B}\right)\right)
$$

for every $A, B \in I F S s(X)$, is an IF-divergence.

This proposition is very useful to prove that some of the most usual functions used for measuring the difference between intuitionistic fuzzy sets are also IF-divergences. Let us see some examples of this application:

Example 3.9 Let us consider a finite universe $X=\left\{x_{1}, \ldots, x_{n}\right\}$, and let $l$ denote the Hamming distance for fuzzy sets, that is defined by:

$$
l(A, B)=\sum_{i=1}^{n}\left|\mu_{A}\left(x_{i}\right)-\mu_{B}\left(x_{i}\right)\right|,
$$

We are going to consider $f_{1}$ and $f_{2}$ two functions defined by:

$$
\begin{aligned}
f_{1}(x, y) & =\frac{x+y}{2}, \\
f_{2}(x, y) & =\max \{x, y\} .
\end{aligned}
$$

It is obvious that these functions fulfill conditions of Proposition 3.8. Then, it holds that the following functions are IFS-divergences:

$$
\begin{aligned}
D_{\mathrm{H}}(A, B)= & f_{1}\left(l\left(\mu_{A}, \mu_{B}\right), l\left(\nu_{A}, \nu_{B}\right)\right) \\
= & \frac{1}{2} \sum_{i=1}^{n}\left(\left|\mu_{A}\left(x_{i}\right)-\mu_{B}\left(x_{i}\right)\right|\right. \\
+ & \left.\left|\nu_{A}\left(x_{i}\right)-\nu_{B}\left(x_{i}\right)\right|\right) . \\
d_{\mathrm{H}}(A, B)= & f_{2}\left(l\left(\mu_{A}, \mu_{B}\right), l\left(\nu_{A}, \nu_{B}\right)\right) \\
= & \sum_{i=1}^{n} \max \left\{\left|\mu_{A}\left(x_{i}\right)-\mu_{B}\left(x_{i}\right)\right|,\right. \\
& \left.\left|\nu_{A}\left(x_{i}\right)-\nu_{B}\left(x_{i}\right)\right|\right\} .
\end{aligned}
$$

In fact, the second one is known as the Hausdorff distance ([11]), and the first one is a function defined by Hong and King ([12]). 
The following result shows us that, if we have an IF-divergence, it is possible to built some other IFdivergences using a nondecreasing function.

Proposition 3.10 Let $D_{\mathrm{IF}}$ be an IF-divergence defined on an universe $X$. If $\varphi$ is a nondecreasing function $\varphi:[0, \infty) \rightarrow[0, \infty), \varphi(0)=0$, then $D_{\mathrm{IF}}^{\varphi}$, defined by:

$$
D_{\mathrm{IF}}^{\varphi}(A, B)=\varphi\left(D_{\mathrm{IF}}(A, B)\right), \text { for } A, B \in I F S s(X),
$$

is other IF-divergence.

This follows directly from the definition of an IFdivergence and properties of $\varphi$.

In the next example we are going to use the previous proposition to prove that some functions are IF-divergences.

Example 3.11 Hung and Yang ([13]) defined the following functions:

$$
\begin{aligned}
D_{\mathrm{HY}}^{1}(A, B) & =d_{\mathrm{H}}(A, B) . \\
D_{\mathrm{HY}}^{2}(A, B) & =\frac{e^{d_{\mathrm{H}}(A, B)}-e^{-1}}{1-e^{-1}} . \\
D_{\mathrm{HY}}^{3}(A, B) & =\frac{1-d_{\mathrm{H}}(A, B)}{1+d_{\mathrm{H}}(A, B)} .
\end{aligned}
$$

We have already proven that the Hausdorff distance is an IF-divergence. Therefore, $D_{\mathrm{HY}}^{1}$ also is an IFdivergence. To prove that $D_{\mathrm{HY}}^{2}$ and $D_{\mathrm{HY}}^{3}$ are $I F$ divergences, it is enough to note that the functions:

$$
\begin{aligned}
\varphi_{1}(t) & =\frac{e^{t}-e^{-1}}{1-e^{-1}}, \\
\varphi_{2}(t) & =\frac{1-t}{1+t},
\end{aligned}
$$

are nondecreasing functions, and therefore applying Proposition 3.10, both functions

$$
\begin{aligned}
& D_{\mathrm{HY}}^{2}(A, B)=\varphi_{1}\left(d_{\mathrm{H}}(A, B)\right), \\
& D_{\mathrm{HY}}^{3}(A, B)=\varphi_{2}\left(d_{\mathrm{H}}(A, B)\right),
\end{aligned}
$$

are IF-divergences.

\section{Examples of IFS-Divergences}

We have already commented that a lot of different types of functions for measuring the difference between intuitionistic fuzzy sets have been presented in the literature. In fact, we are going to present some examples of dissimilarity measures, and we should prove if these functions fulfill or not the axioms presented in Definition 3.1. From now on, we are going to describe some of the more usual dissimilarity measures existing in the literature, explaining if they are or not divergence measures.

Let us consider a finite universe $X=$ $\left\{x_{1}, \ldots, x_{n}\right\}$. First of all, let us recall that the IFdivergences presented in Examples 3.9 and 3.11 are dissimilarity measures. The Hausdorff divergence is one of the most common functions used for measuring the distance between intuitionistic fuzzy sets. Furthermore, this function is a dissimilarity and an IF-divergence measure. Another important dissimilarities are the Hamming distance for intuitionistic fuzzy sets and the normalized Euclidean distance. They are defined by:

- The Hamming distance $([3])$ :

$$
\begin{aligned}
l_{\mathrm{IFS}}(A, B) & =\sum_{i=1}^{n}\left(\left|\mu_{A}\left(x_{i}\right)-\mu_{B}\left(x_{i}\right)\right|\right. \\
& +\left|\nu_{A}\left(x_{i}\right)-\nu_{B}\left(x_{i}\right)\right| \\
& \left.+\left|\pi_{A}\left(x_{i}\right)-\pi_{B}\left(x_{i}\right)\right|\right) .
\end{aligned}
$$

- The normalized Euclidean $([6])$ :

$$
\begin{array}{r}
q_{\mathrm{IFS}}(A, B)=\left(\frac{1}{2 n} \sum_{i=1}^{n}\left(\mu_{A}\left(x_{i}\right)-\mu_{B}\left(x_{i}\right)\right)^{2}\right. \\
+\left(\nu_{A}\left(x_{i}\right)-\nu_{B}\left(x_{i}\right)\right)^{2} \\
\left.+\left(\pi_{A}\left(x_{i}\right)-\pi_{B}\left(x_{i}\right)\right)^{2}\right)^{1 / 2} .
\end{array}
$$

These dissimilarities fulfill the properties of Definition 3.1, and therefore they are also IF-divergences.

Next, we are going to make an overview of other purposed dissimilarity measures we can find in the literature. First of all, Chen $([6,7])$ defined the dissimilarity:

$$
D_{\mathrm{C}}(A, B)=\frac{1}{2 n} \sum_{i=1}^{n}\left|S_{A}\left(x_{i}\right)-S_{B}\left(x_{i}\right)\right|,
$$

where $S_{A}\left(x_{i}\right)=\mu_{A}\left(x_{i}\right)-\nu_{A}\left(x_{i}\right)$ and $S_{B}\left(x_{i}\right)=$ $\mu_{B}\left(x_{i}\right)-\nu_{B}\left(x_{i}\right)$. However, Hung and Kim ([12]) showed that this function is counterintuitive, in the sense that every pair of intuitionistic fuzzy sets $A$ and $B$ such that $S_{A}\left(x_{i}\right)=S_{B}\left(x_{i}\right)$ for every $i=$ $1, \ldots, n, D_{\mathrm{C}}(A, B)=0$. For example, it is enough to consider $X=\{x\}$ and $A=\{(x, 0,0)\}, B=$ $\{(x, 0.5,0.5)\}$. For them it holds that $D_{\mathrm{C}}(A, B)=$ 0 , that is clearly counterintuitive. For this reason, Hong and King ([12]) defined two functions:

$$
\begin{array}{r}
D_{\mathrm{H}}(A, B)=\frac{1}{2 n}\left(\sum_{i=1}^{n}\left|\mu_{A}\left(x_{i}\right)-\mu_{B}\left(x_{i}\right)\right|\right. \\
\left.+\left|\nu_{A}\left(x_{i}\right)-\nu_{B}\left(x_{i}\right)\right|\right) . \\
D_{\mathrm{L}}(A, B)=\frac{1}{4 n}\left(\left(\sum_{i=1}^{n} S_{A}\left(x_{i}\right)-S_{B}\left(x_{i}\right)\right)\right. \\
-\left(\sum_{i=1}^{n}\left|\mu_{A}\left(x_{i}\right)-\mu_{B}\left(x_{i}\right)\right|\right. \\
\left.\left.+\left|\nu_{A}\left(x_{i}\right)-\nu_{B}\left(x_{i}\right)\right|\right)\right) .
\end{array}
$$

These dissimilarities are also an IF-divergence. Another dissimilarity measure that is also an IFdivergence is the one purposed by Li et al. ([18]), $D_{\mathrm{O}}$, and the one defined by Mitchell ([20]), $D_{\mathrm{HB}}$. They are defined by:

$$
\begin{aligned}
D_{\mathrm{O}}(A, B)=\frac{1}{\sqrt{2 n}} & \left(\sum_{i=1}^{n}\left(\mu_{A}\left(x_{i}\right)-\mu_{B}\left(x_{i}\right)\right)^{2}\right. \\
& \left.+\left(\nu_{A}\left(x_{i}\right)-\nu_{B}\left(x_{i}\right)\right)^{2}\right)^{0.5} . \\
D_{\mathrm{HB}}(A, B)=\frac{1}{2 \sqrt[p]{n}} & \left(\left(\sum_{i=1}^{n}\left|\mu_{A}\left(x_{i}\right)-\mu_{B}\left(x_{i}\right)\right|^{p}\right)^{\frac{1}{p}}\right. \\
& \left.+\left(\sum_{i=1}^{n}\left|\nu_{A}\left(x_{i}\right)-\nu_{B}\left(x_{i}\right)\right|^{p}\right)^{\frac{1}{p}}\right) .
\end{aligned}
$$

Dengfeng and Chuntian ([16]) presented a dissimilarity measure that is not IF-divergence.

$$
D_{\mathrm{DC}}(A, B)=\frac{1}{n^{\frac{1}{p}}}\left(\sum_{i=1}^{n}\left|m_{A}\left(x_{i}\right)-m_{B}\left(x_{i}\right)\right|^{p}\right)^{\frac{1}{p}},
$$


where $m_{A}\left(x_{i}\right)=\frac{1}{2}\left(\mu_{A}\left(x_{i}\right)-1+\nu_{A}\left(x_{i}\right)\right.$ and $m_{B}\left(x_{i}\right)=\frac{1}{2}\left(\mu_{B}\left(x_{i}\right)-1+\nu_{B}\left(x_{i}\right)\right.$. Liang and Shi ([19]) defined three dissimilarity measures. Only the third is an IF-divergence.

$$
D_{\mathrm{e}}^{p}(A, B)=\frac{1}{n^{\frac{1}{p}}}\left(\sum_{i=1}^{n}\left(\phi_{\mu A B}\left(x_{i}\right)+\phi_{\nu A B}\left(x_{i}\right)\right)^{p}\right)^{\frac{1}{p}}
$$

where $\phi_{\mu A B}\left(x_{i}\right)=\frac{1}{2}\left|\mu_{A}\left(x_{i}\right)-\mu_{B}\left(x_{i}\right)\right|$ y $\phi_{\nu A B}\left(x_{i}\right)=$ $\frac{1}{2}\left|\nu_{A}\left(x_{i}\right)-\nu_{B}\left(x_{i}\right)\right|$.

$$
D_{\mathrm{s}}^{p}(A, B)=\frac{1}{n^{\frac{1}{p}}}\left(\sum_{i=1}^{n}\left(\varphi_{s 1}\left(x_{i}\right)-\varphi_{s 2}\left(x_{i}\right)\right)^{p}\right)^{\frac{1}{p}}
$$

where

$$
\begin{aligned}
& \varphi_{s 1}\left(x_{i}\right)=\frac{1}{2}\left|m_{A 1}\left(x_{i}\right)-m_{B 1}\left(x_{i}\right)\right| . \\
& \varphi_{s 2}\left(x_{i}\right)=\frac{1}{2}\left|m_{A 2}\left(x_{i}\right)-m_{B 2}\left(x_{i}\right)\right| . \\
& m_{A 1}\left(x_{i}\right)=\frac{1}{2}\left(\mu_{A}\left(x_{i}\right)+m_{A}\left(x_{i}\right)\right) . \\
& m_{A 2}\left(x_{i}\right)=\frac{1}{2}\left(m_{A}\left(x_{i}\right)+1-\nu_{A}\left(x_{i}\right)\right) . \\
& m_{B 1}\left(x_{i}\right)=\frac{1}{2}\left(\mu_{B}\left(x_{i}\right)+m_{B}\left(x_{i}\right)\right) . \\
& m_{B 2}\left(x_{i}\right)=\frac{1}{2}\left(m_{B}\left(x_{i}\right)+1-\nu_{B}\left(x_{i}\right)\right) . \\
& m_{A}\left(x_{i}\right)=\frac{1}{2}\left(\mu_{A}\left(x_{i}\right)+1-\nu_{A}\left(x_{i}\right)\right) . \\
& m_{B}\left(x_{i}\right)=\frac{1}{2}\left(\mu_{B}\left(x_{i}\right)+1-\nu_{B}\left(x_{i}\right)\right) . \\
& D_{\mathrm{h}}^{p}(A, B)=\frac{1}{(3 n)^{1 / p}}\left(\sum _ { i = 1 } ^ { n } \left(\eta_{1}(i)+\eta_{2}(i)\right.\right. \\
& \left.\left.\quad+\eta_{3}(i)\right)^{p}\right)^{1 / p}
\end{aligned}
$$

where

$$
\begin{aligned}
& \eta_{1}(i)=\phi_{\mu}\left(x_{i}\right)+\phi_{\nu}\left(x_{i}\right) . \\
& \eta_{2}(i)=\left|m_{A}\left(x_{i}\right)-m_{B}\left(x_{i}\right)\right| . \\
& \eta_{3}(i)=\max \left(l_{A}(i), l_{B}(i)\right)-\min \left(l_{A}(i), l_{B}(i)\right) . \\
& l_{A}(i)=\frac{1}{2}\left(1-\nu_{A}\left(x_{i}\right)-\mu_{A}\left(x_{i}\right)\right) . \\
& l_{B}(i)=\frac{1}{2}\left(1-\nu_{B}\left(x_{i}\right)-\mu_{B}\left(x_{i}\right)\right) .
\end{aligned}
$$

\begin{tabular}{|c|c|c|c|c|}
\hline Name & Notation & Ax. $1 \& 2$ & Ax. $3 \& 4$ & $\overline{\mathrm{Ax} .5}$ \\
\hline $\begin{array}{c}\text { Normalized } \\
\text { Hammaing }\end{array}$ & $l_{\text {IFS }}$ & OK & $\mathrm{OK}$ & OK \\
\hline Hausdorff & $d_{\mathrm{H}}$ & OK & OK & OK \\
\hline $\begin{array}{l}\text { Normalized } \\
\text { Eucliden }\end{array}$ & $q_{\text {IFS }}$ & OK & $\mathrm{OK}$ & OK \\
\hline Chen & $D_{\mathrm{C}}$ & OK & FAIL & OK \\
\hline $\begin{array}{l}\text { Hong and } \\
\text { Kim (I) }\end{array}$ & $D_{\mathrm{H}}$ & OK & OK & $\mathrm{OK}$ \\
\hline $\begin{array}{l}\text { Hong and } \\
\text { Kim (II) }\end{array}$ & $D_{\mathrm{L}}$ & OK & OK & OK \\
\hline Li et al. & $D_{\mathrm{O}}$ & OK & $\mathrm{OK}$ & OK \\
\hline $\begin{array}{c}\text { Dengfeng } \\
\text { and Chuntian }\end{array}$ & $D_{\mathrm{DC}}$ & OK & FAIL & OK \\
\hline Mitchell & $D_{\mathrm{HB}}$ & OK & FAIL & $\mathrm{OK}$ \\
\hline $\begin{array}{l}\text { Liang and } \\
\text { Shi (I) }\end{array}$ & $D_{\mathrm{e}}^{p}$ & OK & FAIL & OK \\
\hline $\begin{array}{l}\text { Liang and } \\
\text { Shi (II) }\end{array}$ & $D_{\mathrm{S}}^{p}$ & OK & FAIL & OK \\
\hline $\begin{array}{l}\text { Hung and } \\
\text { Yang (I) }\end{array}$ & $D_{\mathrm{HY}}^{1}$ & OK & OK & OK \\
\hline $\begin{array}{l}\text { Hung and } \\
\text { Yang (II) }\end{array}$ & $D_{\mathrm{HY}}^{2}$ & OK & OK & OK \\
\hline $\begin{array}{l}\text { Hung and } \\
\text { Yang (III) }\end{array}$ & $D_{\mathrm{HY}}^{3}$ & OK & OK & OK \\
\hline $\begin{array}{l}\text { Hung and } \\
\text { Yang (IV) }\end{array}$ & $D_{\omega 1}$ & OK & FAIL & OK \\
\hline $\begin{array}{l}\text { Hung and } \\
\text { Yang (V) }\end{array}$ & $D_{\omega 2}$ & OK & FAIL & OK \\
\hline $\begin{array}{l}\text { Hung and } \\
\text { Yang (VI) }\end{array}$ & $D_{\mathrm{pk} 1}$ & OK & FAIL & OK \\
\hline $\begin{array}{l}\text { Hung and } \\
\text { Yang (VII) }\end{array}$ & $D_{\mathrm{pk} 2}$ & OK & FAIL & OK \\
\hline $\begin{array}{l}\text { Hung and } \\
\text { Yang (VIII) }\end{array}$ & $D_{\mathrm{pk} 3}$ & OK & FAIL & OK \\
\hline
\end{tabular}

We have already seen three IF-divergences introduced by Hung and Yang, that were nondecreasing transformations of the Hamming distances. They also purposed other dissimilarity measures ([13]), but they were not IF-divergences.

$$
\begin{aligned}
& D_{\omega 1}(A, B)=1-\frac{1}{n} \sum_{i=1}^{n} \frac{m_{1}\left(x_{i}\right)+m_{2}\left(x_{i}\right)}{M_{1}\left(x_{i}\right)+M_{2}\left(x_{i}\right)} . \\
& D_{\omega 2}(A, B)=1-\frac{1}{n} \sum_{i=1}^{n}\left(1-\frac{1}{2}\left|\mu_{A}\left(x_{i}\right)-\mu_{B}\left(x_{i}\right)\right|\right. \\
& D_{\mathrm{pk} 1}(A, B)=1-\frac{\sum_{i=1}^{n} m_{1}\left(x_{i}\right)+m_{2}\left(x_{i}\right)}{\sum_{i=1}^{n} M_{1}\left(x_{i}\right)+M_{2}\left(x_{i}\right)} . \\
& \left.+\left|\nu_{A}\left(x_{i}\right)-\nu_{B}\left(x_{i}\right)\right|\right) \text {. } \\
& D_{\mathrm{pk} 2}(A, B)=\frac{\sum_{i=1}^{n}\left|\mu_{A}\left(x_{i}\right)-\mu_{B}\left(x_{i}\right)\right|+\nu_{A}\left(x_{i}\right)-\nu_{B}\left(x_{i}\right) \mid}{\sum_{i=1}^{n}\left|\mu_{A}\left(x_{i}\right)+\mu_{B}\left(x_{i}\right)\right|+\nu_{A}\left(x_{i}\right)+\nu_{B}\left(x_{i}\right) \mid} .
\end{aligned}
$$

where

$$
\begin{aligned}
& m_{1}\left(x_{i}\right)=\min \left\{\mu_{A}\left(x_{i}\right), \mu_{B}\left(x_{i}\right)\right\} \\
& m_{2}\left(x_{i}\right)=\min \left\{\nu_{A}\left(x_{i}\right), \nu_{B}\left(x_{i}\right)\right\} \\
& M_{1}\left(x_{i}\right)=\max \left\{\mu_{A}\left(x_{i}\right), \mu_{B}\left(x_{i}\right)\right\} \\
& M_{2}\left(x_{i}\right)=\max \left\{\nu_{A}\left(x_{i}\right), \nu_{B}\left(x_{i}\right)\right\}
\end{aligned}
$$

In Table 1 we can see a summary of the functions we have seen in this section. There, we can see which properties fulfill every function. We have to note that all this function fulfill axiom 5 , and therefore axioms 3 and 4 are equivalents by Proposition 3.5.
Table 1: Examples of dissimilarities.

\section{Concluding remarks}

In this work we have presented an axiomatic definition of divergences for measuring the difference between intuitionistc fuzzy sets. We have studied the relationship among IF-divergences, distances and dissimilarities, and we have concluded that the axiomatic of IF-divergence is more restrictive than the one of dissimilarities. Finally, we have proved that some of the most usual dissimilarities used in the literature are also IF-divergences, and we provided some methods for building IF-divergences. We have to recall that this is just a first approach to our objective of building an axiomatic theory of the divergences for intuitionistic fuzzy sets. In fact, in future work we hope to present a generalization of the notion of "local divergence" for intuitionistic fuzzy sets. We also want to formulate a more general theory involving distances, dissimilarities and IFdivergences, as we can see in the work of Bouchon et al. ([4]). The application of these studies for multiattribute decision making models and pattern recognition problems is our last open problem in relation to this work. These application will be done in base on some applied papers based on particular examples of divergence measures. More precisely, we will complete in a general context the use of these measures in pattern recognition made by $[13,17,19]$ and the use in decision making made by $[23,24]$.

\section{Acknowledgement}

The research in this paper is partly supported by the Internationalization Plan 2010 of the University of Oviedo, the Science and Education Ministry FPU grant AP2009-1034, by the Agency of the Slovak Ministry of Education for the Structural Funds of 
the EU, under project ITMS:26220120007 and the Spanish Ministry of Science and Innovation grant MTM2010-17844.

\section{References}

[1] K. Atanassov, Intuitionistic Fuzzy Sets. In proceedings of VII ITKR, Sofia, 1983.

[2] K. Atanassov, Intuitionistic Fuzzy Sets, Fuzzy Sets and Systems, 20:87-96, Elsevier, 1986.

[3] K. Atanassov, Intuitionistic Fuzzy Sets: Theory and Applications, Physica-Verlang, Wyrzburg, 1999.

[4] B. Bouchon-Meunier, M. Rifqi and S. Bothorel, Towards general measures of comparison of objects, Fuzzy Sets and Systems, 84:143-153, Elsevier, 1996.

[5] H. Bustince and P. Burrillo, Vague sets are intuitionistic fuzzy sets, Fuzzy sets and Systems, 19(3):403-405, Elsevier, 1996.

[6] S. M. Chen, Measures of similarity between vague sets, Fuzzy Sets and Systems, 74(2):217223, Elsevier, 1995.

[7] S. M. Chen, Similarity measures between vague sets and between elements, IEEE Trans. Syst. Mn Cybernet., 27(1):153-158, 1997.

[8] I. Couso, L. Garrido, S. Montes and L. Sánchez, Expected Pair-Wise Comparison of the Outcomes of a Fuzzy Random Variable. In C. Borgelt, G. Gonzï $₫ \frac{1}{2}$ lez-Rodrï $₫ \frac{1}{2}$ guez, W. Trutschnig, M. A. Lubiano, M. A. Gil, P. Grzeforzewski and O. Hryniewicz, editors, proceeding of the Soft Methods in Probability and Statistics ( SMPS 2010), Advances in Intelligent and Soft Computing 77, pages 105-113, 2010.

[9] D. Dubois, S. Gottwald, P. Hajek, J. Kacprzyk and H. Prade, Terminological difficulties in fuzzy set theory-The case of "Intuitionistic Fuzzy Sets", Fuzzy Sets and Systems, 156(3):485-491, Elsevier, 2005.

[10] G. Deschrijver and E. Kerre, A generalization of operators on intuitionistic fuzzy sets using triangular norms and conorms, Notes on IFS, 1:19-27, 2002.

[11] P. Grzegorzewski, Distances between intuitionistic fuzzy sets and/or interval-valued fuzzy sets based on the Hausdorff metric, Fuzzy Sets and Systems, 148:319-328, Elsevier, 2004.

[12] D. H. Hong and C. Kim, A note on similarity measures between vague sets and between elements, Information Sciences, 115:83-96, Elsevier, 1999.

[13] W. L. Hung and M. S Yang, Similarity measures of intuitionistic fuzzy sets based on Hausdorff distance, Pattern recognition Letters, 25: 1603-1611, Elsevier, 2004.

[14] W. L. Hung and M. S Yang, Similarity measures of intuitionistic fuzzy sets based on $L_{p}$ metric, Int. Journal of Approximate Reasoning, 46:120-136, Elsevier, 2007.

[15] V. Janiš, t-norm based cuts of intuitionistic fuzzy sets, Information Sciences, 180(7):11341137, Elsevier, 2010.

[16] D. F. Li and C. T. Cheng, New similarity measures of intuitionistic fuzzy sets and application to pattern recognitions, Pattern recognition Letters, 23:221-225, Elsevier, 2002.

[17] Y. Li, D. L. Olson and Z. Qin, Similarity measures between intuitionistic fuzzy (vague) sets: A comparative analysis, Pattern recognition Letters, 28:278-285, Elsevier, 2007.

[18] D. F. Li, C. Zhongxian and Y. Degin, Similarity measures between vague sets and vague entropy, J. Computer Sciences, 29(12):129-132, Elsevier, 2002.

[19] Z. Liang and P. Shi, Similarity measures on intuitionistic fuzzy sets, Pattern recognition Letters, 24:2687-2693, Elsevier, 2003.

[20] H. B. Mitchell, On the Dengfeng-Chuntian similarity measure and its application to pattern recognition, Pattern recognition Letters, 24:3101-3104, Elsevier, 2003.

[21] S. Montes, I. Couso, P. Gil and C. Bertoluzza, Divergence measure between fuzzy sets, International Journal of Approximate Reasoning, 30:91-105, Elsevier, 2002.

[22] E. Szmidt and J. Kacprzyk, Analysis of Similarity Measures for Atanassov's Intuitionistic Fuzzy Sets. In J. P. Carvalho, D. Dubois, U. Kaymak and J. M. C. Sousa, editors, proceedings of the International Fuzzy Systems Association World Congress and 2009 European Society of Fuzzy Logic and Technology Conference (IFSA-EUSFLAT 2009), 1416-1421, 2009.

[23] E. Szmidt and J. Kacprzyk, Distances between intuitionistic fuzzy sets, Fuzzy sets and systems, 114:505-518, Elsevier, 2000.

[24] Z. Xu, Some similarity measures of intuitionistic fuzzy sets and their applications to multiple attribute decision making, Fuzzy Optim. Decis. Making, 6:109-121, Springer, 2007.

[25] L. A. Zadeh, Fuzzy sets, Information Control, 8:338-353, 1965. 